\title{
A Model Theoretic Proof of Completeness of an Axiomatization of Monadic Second-Order Logic on Infinite Words
}

\author{
Colin Riba* \\ ENS de Lyon, Université de Lyon, LIP \\ colin.riba@ens-lyon.fr \\ http://perso.ens-lyon.fr/colin.riba/
}

\begin{abstract}
We discuss a complete axiomatization of Monadic SecondOrder Logic (MSO) on infinite words.By using model-theoretic methods, we give an alternative proof of D. Siefkes' result that a fragment with full comprehension and induction of second-order Peano's arithmetic is complete w.r.t. the validity of MSO-formulas on infinite words. We rely on Feferman-Vaught Theorems and the Ehrenfeucht-Fraïssé method for Henkin models of MSO. Our main technical contribution is an infinitary Feferman-Vaught Fusion of such models. We show it using Ramseyan factorizations similar to those for standard infinite words.
\end{abstract}

\section{Introduction}

We discuss the completeness of an axiomatization of Monadic Second-Order Logic (MSO) on infinite words. MSO on infinite words is known to be decidable since the celebrated work of Büchi [2]. The usual route is to translate MSOformulas to finite state automata running on infinite words. Such automata provide an established framework for the specification and verification of nonterminating programs, while MSO is a yardstick language for expressing properties about them. We refer to e.g. $7[6] 8$ for comprehensive presentations of the subject.

D. Siefkes has shown in [1] that a fragment of second-order Peano's arithmetic containing the comprehension axiom scheme and the induction axiom is complete with respect to the standard model: every MSO-formula true on infinite words is provable. The approach taken there was to formalize the translation of MSOformulas to Büchi automata. This requires to represent automata in the logic and to formalize the correctness proof of the translation in the corresponding deduction system.

In this paper, we give an alternative proof of Siefkes' completeness result by using model-theoretic tools. This leads to a more abstract proof which does not require explicit manipulation of automata in the logic. To our knowledge, such approaches to MSO have not been much explored compared to the great body of work on automata and corresponding algebraic structures [68].

* UMR 5668 CNRS ENS Lyon UCBL INRIA. 
We follow the method of [5], where complete axiomatizations of variants of MSO on finite trees are presented. Starting from Henkin completeness, we show that all models of our axiomatization are equivalent w.r.t. the validity of MSO-formulas. As in [5], we use Feferman-Vaught Theorems obtained by the Ehrenfeucht-Fraïssé method [10].

In contrast with [107], works like [5] or the present one have to handle nonstandards models of second-order arithmetic. As far as Henkin completeness is concerned, a model $\mathcal{M}$ of MSO can be seen as a structure with two domains: a domain $\mathcal{M}^{\iota}$ of individuals and a domain $\mathcal{M}^{o} \subseteq \mathcal{P}\left(\mathcal{M}^{\iota}\right)$ of sets of individuals (called predicates in this paper). Besides non-standards individuals (whose order type is very different from $\omega$ ), the main difficulty is that $\mathcal{M}^{o}$ is in general strictly contained in $\mathcal{P}\left(\mathcal{M}^{\iota}\right)$ : there might not be "enough" predicates.

A crucial observation due to K. Doets [3] makes apparent in (possibly nonstandard) models a structure similar to standard infinite words. Our main technical contribution is a kind of Feferman-Vaught Infinitary Fusion for such models. Intuitively, it is a model-theoretic counterpart to a run of a Büchi automaton on a standard infinite word. The point is to ensure that such a "run" always exists as a predicate of a given model. For this, we use Ramseyan factorizations similar to those of infinite words (see e.g. [8]).

The paper is organized as follows. In Section 2 we describe our formal system for MSO, as well as the class of models we are interested in. These models are motivated by usual results on Henkin completeness for second-order logic that we briefly recall. We present in Section 3 the notions on the Ehrenfeucht-Fraïssé method that we will need. We use it to prove a Feferman-Vaught Finite Sums Lemma for linearly ordered structures with parameters, which is discussed in Section 4. We then give the main argument for completeness in Section 5] It relies on an infinitary version of the Finite Sums Lemma, that we call "Infinite Fusion" and which is shown in Section 6 .

A full version of this paper is available on the author's web page

http://perso.ens-lyon.fr/colin.riba/papers/msofull.pdf.

\section{A Deduction System for Monadic Second-Order Logic on Infinite Words}

\section{$2.1 \quad$ Language}

We consider a formulation of Monadic Second-Order Logic (MSO) based on a two-sorted language: There is one sort $\iota$ intended to range over individuals and one sort $o$ intended to range over monadic (or one-place) predicates on individuals. We assume given two countable sets $\mathcal{V}_{\iota}=\{x, y, z, \ldots\}$ and $\mathcal{V}_{o}=$ $\{X, Y, Z, \ldots\}$ of respectively individual and predicate variables. The formulas of MSO are then defined by the following grammar:

$$
\phi, \psi \in \Lambda \quad:=X x \quad|x<y \quad| \neg \phi \quad|\quad \phi \vee \psi| \exists X \phi \mid \exists x \phi
$$

The set $\mathrm{FV}(\phi)$ of free (individual and predicate) variables of a formula $\phi$ is defined as usual. A sentence (or closed formula) is a formula with no free variable, 
i.e. a formula $\phi$ such that $\mathrm{FV}(\phi)=\emptyset$. Formulas are identified modulo renaming of their bound variables. The capture-avoiding substitution of $y$ for $x$ in $\phi$ is written $\phi[y / x]$.

Note that there is no primitive equality in $\Lambda$. This is discussed in Section 2.4 The other logical connectives are defined as usual:

$$
\begin{aligned}
& \phi \rightarrow \psi \quad:=\neg \phi \vee \psi \\
& \phi \wedge \psi \quad:=\neg(\neg \phi \vee \neg \psi) \\
& \phi \longleftrightarrow \psi:=(\phi \rightarrow \psi) \wedge(\psi \rightarrow \phi) \\
& \begin{array}{l}
\forall X \phi:=\neg \exists X \neg \phi \\
\forall x \phi:=\neg \exists x \neg \phi
\end{array}
\end{aligned}
$$

\subsection{Deduction for Second-Order Logic}

We now discuss formal deduction for second-order logic. As usual, the rules for second-order logic are those of the (two-sorted) classical predicate calculus together with the comprehension axiom scheme (see e.g. 9]). There are several different formulations equivalent w.r.t. provability. The following Natural Deduction system is a possible choice.

The deduction relation is writen $\Gamma \vdash \phi$, where $\Gamma$ is a (possibly empty) finite unordered list of (possibly not closed) formula, and $\phi$ is a (possibly not closed) formula. It is inductively defined by the following rules.

- Rules for propositional logic:

$$
\begin{array}{ccc}
\frac{}{\Gamma \vdash \phi \vee \neg \phi} & \frac{\Gamma \vdash \phi \quad \Gamma \vdash \neg \phi}{\Gamma \vdash \psi} \\
\frac{\Gamma \vdash \phi}{\Gamma \vdash \phi \vee \psi} & \frac{\Gamma \vdash \psi}{\Gamma \vdash \phi \vee \psi} & \frac{\Gamma \vdash \phi \vee \psi}{\Gamma \vdash \phi} \quad \Gamma \vdash \varphi \quad \Gamma, \psi \vdash \varphi \\
\Gamma \vdash \varphi
\end{array}
$$

- Rules for predicate logic (where $\mathcal{X}, \mathcal{Y} \in \mathcal{V}_{\iota}$ or $\mathcal{X}, \mathcal{Y} \in \mathcal{V}_{o}$ ):

$$
\frac{\Gamma \vdash \phi[\mathcal{Y} / \mathcal{X}]}{\Gamma \vdash \exists \mathcal{X} \phi} \quad \frac{\Gamma \vdash \exists \mathcal{X} \phi \quad \Gamma, \phi \vdash \psi}{\Gamma \vdash \psi}(\mathcal{X} \notin \operatorname{FV}(\Gamma, \psi))
$$

- Comprehension scheme (for all formula $\phi$ ):

$$
\overline{\Gamma \vdash \exists X \forall x(X x \longleftrightarrow \phi)}(X \notin \mathrm{FV}(\phi))
$$

\subsection{Models of Second-Order Logic}

We discuss the class of structures (or models) we will use to interpret the language of MSO presented in Section 2.1. These structures are motivated by known results on Henkin completeness that we briefly recall. 
Structures, Assignments and Satisfiability. We consider (Henkin) structures $\mathcal{M}$ of the form $\left(\mathcal{M}^{\iota}, \mathcal{M}^{o},<_{\mathcal{M}}\right)$ where $\mathcal{M}^{\iota}$ is a non-empty set of individuals, $\mathcal{M}^{o} \subseteq$ $\mathcal{P}\left(\mathcal{M}^{\iota}\right)$ is a non-empty set of predicates and $<_{\mathcal{M}}$ is a binary relation on $\mathcal{M}^{\iota}$. We call $\mathcal{M}^{\iota}$ and $\mathcal{M}^{o}$ respectively the individual and predicate domains of $\mathcal{M}$.

An $\mathcal{M}$-assignment is a map $\rho:\left(\mathcal{V}_{\iota} \cup \mathcal{V}_{o}\right) \rightarrow\left(\mathcal{M}^{\iota} \cup \mathcal{M}^{o}\right)$ which respects the sorts, i.e. such that $\rho(x) \in \mathcal{M}^{\iota}$ and $\rho(X) \in \mathcal{M}^{o}$ if $x \in \mathcal{V}_{\iota}$ and $X \in \mathcal{V}_{o}$. Given $x \in \mathcal{V}_{\iota}$ and $a \in \mathcal{M}^{\iota}$, we write $\rho[a / x]$ for the assignment which maps $x$ to $a$ and is equal to $\rho$ everywhere else. The assignment $\rho[A / X]$ (where $X \in \mathcal{V}_{o}$ and $A \in \mathcal{M}^{o}$ ) is defined similarly.

Given a structure $\mathcal{M}$, an $\mathcal{M}$-assignment $\rho$ and a formula $\phi$, we define the satisfaction relation $\mathcal{M}, \rho \models \phi$ by induction on $\phi$ as usual:

$$
\begin{array}{lll}
\mathcal{M}, \rho \models X x & \text { iff } & \rho(x) \in \rho(X) \\
\mathcal{M}, \rho \models x<y & \text { iff } & \rho(x)<\mathcal{M} \rho(y) \\
\mathcal{M}, \rho \models \neg \phi & \text { iff } & \mathcal{M}, \rho \not \models \phi \\
\mathcal{M}, \rho \models \phi \vee \psi & \text { iff } & \mathcal{M}, \rho \models \phi \text { or } \mathcal{M}, \rho \models \psi \\
\mathcal{M}, \rho \models \exists X \phi & \text { iff } & \text { there is some } A \in \mathcal{M}^{\circ} \text { such that } \mathcal{M}, \rho[A / X]=\phi \\
\mathcal{M}, \rho \models \exists x \phi & \text { iff } & \text { there is some } a \in \mathcal{M}^{\iota} \text { such that } \mathcal{M}, \rho[a / x] \models \phi
\end{array}
$$

We say that $\phi$ is valid in $\mathcal{M}$ (notation $\mathcal{M} \models \phi$ ) if $\mathcal{M}, \rho \models \phi$ for every $\rho$. A set of formulas $\Delta$ is valid in $\mathcal{M}$ (notation $\mathcal{M} \models \Delta$ ) if $\mathcal{M} \models \phi$ for every $\phi \in \Delta$.

It is sometimes convenient to consider formulas with a fixed assignment of their free variables to some structure $\mathcal{M}$. These formulas are called formulas with parameters in $\mathcal{M}$. We define them as pairs of a formula $\phi$ and a finite partial $\mathcal{M}$-assignment $\nu:\left(\mathcal{V}_{\iota} \cup \mathcal{V}_{o}\right) \rightarrow\left(\mathcal{M}^{\iota} \cup \mathcal{M}^{o}\right)$. The set of free variables of the formula with parameters $(\phi, \nu)$ is $\operatorname{FV}(\phi, \nu):=\mathrm{FV}(\phi) \backslash \operatorname{dom}(\nu)$. We will often write $\phi[\nu(\mathcal{X}) / \mathcal{X} \mid \mathcal{X} \in \operatorname{dom}(\nu)]$ for the formula with parameters $(\phi, \nu)$.

The satisfaction of a formula with parameters $(\phi, \nu)$ in a structure $\mathcal{M}$ and assignment $\rho$ (notation $\mathcal{M}, \rho \models(\phi, \nu)$ ) is defined as the satisfaction of $\phi$ in $\mathcal{M}$ and assignment $\rho[\nu(\mathcal{X}) / \mathcal{X} \mid \mathcal{X} \in \operatorname{dom}(\nu)]$. The corresponding validity relation $\mathcal{M} \models(\phi, \nu)$ holds if $\mathcal{M}, \rho \models(\phi, \nu)$ for every $\rho$.

Second-Order Henkin Structures. Deduction without the comprehension scheme is correct in any structure $\mathcal{M}$ : if $\vdash \phi$ is derivable without using the comprehension then $\phi$ is valid in $\mathcal{M}$. The following notions are useful to handle the comprehension scheme. A set of individuals $A \in \mathcal{P}\left(\mathcal{M}^{\iota}\right)$ is definable if there is a formula $\phi$ and an $\mathcal{M}$-assignment $\rho$ such that

$$
A=\left\{a \in \mathcal{M}^{\iota} \mid \mathcal{M}, \rho[a / x] \models \phi\right\}
$$

Of course, all $A \in \mathcal{M}^{o}$ are definable. The converse is more interesting, since $\mathcal{M}$ satisfies every instance of the comprehension scheme if and only if $\mathcal{M}^{o}$ is the set of all definable $A \in \mathcal{P}\left(\mathcal{M}^{\iota}\right)$. In this case, we call $\mathcal{M}$ a second-order (Henkin) structure.

Remark 2.1. (i) We say that $\mathcal{M}$ is full if $\mathcal{M}^{o}=\mathcal{P}\left(\mathcal{M}^{\iota}\right)$. Full structures are second-order. 
(ii) Finite boolean combinations of definable predicates are definable. Hence, the predicate domain of a second-order structure is closed under finite boolean operations.

Henkin Completeness. Usual Henkin completeness holds for deduction w.r.t. validity in all second-order Henkin structures (see e.g. [9]):

Theorem 2.2 (Henkin Completeness). Let $\Delta$ be a set of sentences and $\phi$ be a sentence. Assume that for all second-order Henkin structure $\mathcal{M}$, if $\mathcal{M} \models \Delta$ then $\mathcal{M}=\phi$. Then there is a finite set $\Gamma \subseteq \Delta$ such that $\Gamma \vdash \phi$.

\subsection{Equality}

Monadic Second-Order Logic has a definable equality (see e.g. 9]):

$$
(x \doteq y):=\forall X(X x \rightarrow X y)
$$

Thanks to the comprehension scheme, it is an equivalence relation which moreover satisfies Leibniz's scheme:

$$
\begin{gathered}
\vdash \forall x(x \doteq x) \quad \vdash \forall x y(x \doteq y \rightarrow y \doteq x) \quad \vdash \forall x y z(x \doteq y \rightarrow y \doteq z \rightarrow x \doteq z) \\
\vdash \forall x y(x \doteq y \rightarrow \phi[x / z] \rightarrow \phi[y / z]) \quad(\text { for all formula } \phi)
\end{gathered}
$$

Remark 2.3. Given a second-order structure $\mathcal{M}$, we have $\mathcal{M}^{\iota}, \emptyset \in \mathcal{M}^{o}$ since $\mathcal{M}^{\iota}$ is definable by the formula $(x \doteq x)$.

Second-Order Structures with Correct Equality. It is well-known that the equality $\doteq$ may not be correct: Given a structure $\mathcal{M}$, it is possible that $\mathcal{M} \models(a \doteq b)$ but $a \neq b$, even if $\mathcal{M}$ is second-order (see e.g. [9]). We say that a structure $\mathcal{M}$ has correct equality if $\mathcal{M} \models(a \doteq b)$ implies $a=b$ for all $a, b \in \mathcal{M}^{\iota}$.

Remark 2.4. (i) Full structures have correct equality.

(ii) Consider an arbitrary structure $\mathcal{M}$ with correct equality. Note that every singleton $\{a\}$ with $a \in \mathcal{M}^{\iota}$ is definable (by the formula with parameters $(x \doteq y,[a / x]))$. According to Remark 2.1, (iii), it follows that if $\mathcal{M}$ is secondorder, then $\mathcal{M}^{o}$ contains all the finite subsets of $\mathcal{M}^{\iota}$.

In particular, finite second-order structures with correct equality are full.

As far as Henkin completeness is concerned, it is always possible to assume that a second-order structure has correct equality. We in fact have the following strengthening of Henkin completeness (see e.g. 9]):

Corollary 2.5. Let $\Delta$ be a set of sentences and $\phi$ be a sentence. Assume that for all second-order Henkin structure $\mathcal{M}$ with correct equality, if $\mathcal{M} \models \Delta$ then $\mathcal{M} \models \phi$. Then there is a finite set $\Gamma \subseteq \Delta$ such that $\Gamma \vdash \phi$. 


\subsection{Axiomatization}

The standard model is $\mathrm{N}:=\left(\mathbb{N}, \mathcal{P}(\mathbb{N}),<_{\mathbb{N}}\right)$, where $<_{\mathbb{N}}$ is the usual order on natural numbers. Recall that thanks to the celebrated result of Büchi [2], the monadic theory of $\mathrm{N}$ (i.e. the set of sentences $\phi$ such that $\mathrm{N} \models \phi$ ) is decidable.

In this section, we describe a set $\mathrm{MSO}^{\omega}$ of sentences which completely axiomatizes the monadic theory of $\mathrm{N}$ : for all sentence $\phi$, if $\mathrm{N} \models \phi$ then $\mathrm{MSO}^{\omega} \vdash \phi$. The axiomatization we consider is an adaptation of that of [1] to the language of MSO presented in Section 2.1. This is essentially a fragment of second-order Peano's arithmetic with full comprehension and induction.

For the completeness proof of $\mathrm{MSO}^{\omega}$, we shall also discuss variations on Ramsey's theorem and the axiom of choice in Sections 5 and 6 .

Definition 2.6 $\left(\mathrm{MSO}^{\omega}\right)$. MSO ${ }^{\omega}$ is the set of the following sentences:

- Linear Order axioms:

$$
\begin{gathered}
\forall x \neg(x<x) \quad \forall x y z(x<y \rightarrow y<z \rightarrow x<z) \\
\forall x y(x<y \vee x \doteq y \vee y<x)
\end{gathered}
$$

- Unboundedness axiom:

$$
\forall x \exists y(x<y)
$$

- Induction axiom:

$$
\forall X[\forall x(\forall y(y<x \rightarrow X y) \rightarrow X x) \rightarrow \forall x X x]
$$

- Predecessor axiom:

$$
\forall x(\exists y(y<x) \rightarrow \exists y[y<x \wedge \neg \exists z(y<z \wedge z<x)])
$$

A formula $\phi$ is derivable in $\mathrm{MSO}^{\omega}$ if $\mathrm{MSO}^{\omega} \vdash \phi$ is derivable using the deduction system of Section 2.2.

$A$ second-order structure with correct equality $\mathcal{M}$ is a model of $\mathrm{MSO}^{\omega}$ if $\mathcal{M} \models$ $\mathrm{MSO}^{\omega}$.

In this paper, we give a model-theoretic proof of Siefkes' completeness result:

Theorem 2.7 (Completeness of $\mathbf{M S O}^{\omega}[11]$ ). For all sentence $\phi$, if $\mathrm{N}=\phi$ then $\mathrm{MSO}^{\omega} \vdash \phi$.

Following the method of [5], our route to Theorem 2.7] is to use usual Henkin completeness (as formulated in Corollary 2.5), and to show that all models of $\mathrm{MSO}^{\omega}$ are equivalent w.r.t. the validity of MSO-formulas. This is the main result of the paper.

Theorem 2.8 (Main Theorem). Let $\mathcal{M}$ be a model of $M^{\prime} O^{\omega}$ and $\phi$ be a sentence. We have $\mathcal{M}=\phi$ if and only if $\mathrm{N} \models \phi$.

Theorem 2.8 is proved in Section[5] As [5], we rely on Feferman-Vaught Theorems proved by the Ehrenfeucht-Fraïssé method.

We now discuss some aspects of the different axioms of $\mathrm{MSO}^{\omega}$. All structures considered here are second-order and have correct equality. 
Orders. We use the following defined formula:

$$
x \leq y:=x<y \vee x \doteq y
$$

Hence, in a structure $\mathcal{M}$ with correct equality, given $a, b \in \mathcal{M}^{\iota}$ we have $\mathcal{M} \models$ $a \leq b$ if and only if $\left(a=b\right.$ or $\left.a<_{\mathcal{M}} b\right)$.

A structure $\mathcal{M}$ is linearly ordered if is satisfies the Linear Order axioms. The first two sentences say that $<_{\mathcal{M}}$ is strict and transitive. Note that $<_{\mathcal{M}}$ is thus antisymmetric: if $a<_{\mathcal{M}} b$ then $b \nless_{\mathcal{M}} a$. The third sentence says that $<_{\mathcal{M}}$ is total. Since $\mathcal{M}$ is assumed to have correct equality, it is equivalent to requiring that for all $a, b \in \mathcal{M}^{\iota}$ we have either $a<_{\mathcal{M}} b$ or $a=b$ or $b<_{\mathcal{M}} a$.

Induction. The induction axiom holds in the standard model $\mathrm{N}$ but is false for instance in the full structure of real numbers 1

Assume that $\mathcal{M}$ satisfies the induction axiom. The contrapositive of induction says that each non-empty predicate $A \in \mathcal{M}^{\circ}$ has minimal elements. If moreover $\mathcal{M}$ is linearly ordered, then $A$ has a unique least element.

Successors and Predecessors. If $\mathcal{M}$ is linearly ordered and satisfies the induction axiom, then every $a \in \mathcal{M}^{\iota}$ which is not maximal has a successor, i.e. there is a unique least $b>_{\mathcal{M}} a$. However, a non minimal $a \in \mathcal{M}^{\iota}$ may not have a predecessor, i.e. a greatest $b<_{\mathcal{M}} a\lfloor$ The predecessor axiom ensures that every non-minimal individual has a predecessor.

Unboundedness. The axiom of Unboundedness is a kind of infinity axiom. Given a structure $\mathcal{M}$, we say that $U \in \mathcal{M}^{\circ}$ is unbounded in $\mathcal{M}$ if for all $a \in \mathcal{M}$ there is some $b \in U$ such that $a<_{\mathcal{M}} b$. If $<_{\mathcal{M}}$ is strict and transitive, then $U$ must be infinite. Note however that the converse does not hold, even for models of $\mathrm{MSO}^{\omega}$.

Remark 2.9 (Non-Standard Models of $\mathrm{MSO}^{\omega}$ ). A model $\mathcal{M}$ of $\mathrm{MSO}^{\omega}$ can be non-standard (i.e. non-isomorphic to the standard model $\mathrm{N}$ ) for two reasons: (i) because its predicate domain $\mathcal{M}^{o}$ is different from $\mathcal{P}\left(\mathcal{M}^{\iota}\right)$ or (ii) because its individual domain is not isomorphic to $\mathbb{N}$. Let us discuss these two points in view of Theorem 2.8

(i) It is well-known that if $\mathcal{M}$ is full (i.e. $\mathcal{M}^{o}=\mathcal{P}\left(\mathcal{M}^{\iota}\right)$ ), then $\mathcal{M}^{\iota}$ is isomorphic to $\mathbb{N}$ (see e.g. 9]). Hence non-standard models $\mathcal{M}$ have $\mathcal{M}^{o} \subsetneq \mathcal{P}\left(\mathcal{M}^{\iota}\right)$.

(ii) Thanks to the Löwenheim-Skolem Theorem (see e.g. [1]), we can always assume that an $\mathrm{MSO}^{\omega}$-model $\mathcal{M}$ has a countable individual domain $\mathcal{M}^{\iota}$. However, the order structure of $\mathcal{M}$ can be very different from that of $\mathbb{N}$. For instance, if $\mathcal{M}$ is a non-standard model of second-order Peano's arithmetic, then it is also a model of $\mathrm{MSO}^{\omega}$. But $\mathcal{M}$ is also a non-standard model of First-Order Peano's Arithmetic, and it is well-known (see e.g. 1]) that its order type is that of: $\mathbb{N}$ followed by $\mathbb{Q}$ copies of $\mathbb{Z}$. In particular, segments of the form $[a, b)=\left\{c \in \mathcal{M}^{\iota} \mid a \leq_{\mathcal{M}} c<\mathcal{M} b\right\}$ may be infinite.

\footnotetext{
${ }^{1}$ The monadic theory of $\mathbb{R}$ is undecidable (see [7] for references).

${ }^{2}$ Besides completeness w.r.t. $\mathrm{N}$, recall that the monadic theory of the ordinal $\omega_{2}$ is independent from ZFC (see [10]).
} 


\section{The Ehrenfeucht-Fraïssé Method}

We present the notions on the Ehrenfeucht-Fraïssé method that we will need. They are mostly variations on those used in [5]. See 4 for a standard reference.

For the remaining of the paper, we fix enumerations of the individual and predicate variables. Let $\mathcal{V}_{\iota}=\left\{x_{1}, \ldots, x_{p}, \ldots\right\}$ and $\mathcal{V}_{o}=\left\{X_{1}, \ldots, X_{q}, \ldots\right\}$. We say that $\phi$ is a $p$-q-formula if $\mathrm{FV}(\phi) \subseteq\left\{x_{1}, \ldots, x_{p}, X_{1}, \ldots, X_{q}\right\}$.

Unlike the rest of the paper, the results discussed in this section are insensitive on whether we are dealing with Henkin structures, general models, or secondorder version thereof. For convenience, we will only consider Henkin structures which are not necessarily second-order. In this context, two formulas $\phi$ and $\psi$ are logically equivalent if $(\phi \longleftrightarrow \psi)$ is valid in all such structures.

\subsection{Logical Equivalence Up to Bounded Quantifier Depth}

The first step is to classify formulas according to their quantifier-depth.

Definition 3.1 (Quantifier-Depth). The quantifier depth $\mathrm{qd}(\phi)$ of a formula $\phi$ is defined by induction on $\phi$ as follows:

$$
\begin{aligned}
\operatorname{qd}(X x) & :=0 & \operatorname{qd}(\exists x \phi) & :=\operatorname{qd}(\phi)+1 \\
\operatorname{qd}(x<y) & :=0 & \operatorname{qd}(\exists X \phi) & :=\operatorname{qd}(\phi)+1 \\
\operatorname{qd}(\neg \phi) & :=\operatorname{qd}(\phi) & \operatorname{qd}(\phi \vee \psi) & :=\max (\operatorname{qd}(\phi), \operatorname{qd}(\psi))
\end{aligned}
$$

We let $\Lambda_{n}^{p, q}$ be the set of $p$-q-formulas of q.d. $\leq n$ and write $\Lambda_{n}$ for $\Lambda_{n}^{0,0}$.

A remarkable property of languages without function symbols, such as the language of MSO, is the following standard observation (see e.g. 4]).

Lemma 3.2 (Finiteness Lemma). Up to logical equivalence, there are only finitely many $p$-q-formulas of quantifier depth $\leq n$.

Recall that logical equivalence is defined as validity of equivalence in all (possibly non second-order) structures. Requiring instead validity of equivalence in all second-order structures has no impact on finiteness: This amounts to add the comprehension axiom scheme, and adding axioms can only reduce the number of equivalence classes.

\subsection{Structures with Parameters}

A structure with parameters is a structure $\mathcal{M}$ together with $a_{1}, \ldots, a_{p} \in \mathcal{M}^{\iota}$ and $A_{1}, \ldots, A_{q} \in \mathcal{M}^{o}$. We write $\bar{a}$ for a finite sequence of individuals of length $|\bar{a}|$, and similarly for $\bar{A}$. If $|\bar{a}|=p$ and $|\bar{A}|=q$ then we say that $(\mathcal{M}, \bar{a}, \bar{A})$ is a $p$-q-structure.

If $\phi$ is a $p$-formula, we write $(\mathcal{M}, \bar{a}, \bar{A}) \models \phi$ for $\mathcal{M} \models \phi[\bar{a} / \bar{x}][\bar{A} / \bar{X}]$. Two $p$ - $q$-structures $(\mathcal{M}, \bar{a}, \bar{A})$ and $(\mathcal{N}, \bar{b}, \bar{B})$ are $n$-equivalent (notation $\left.\equiv_{n}^{p, q}\right)$ if they satisfy the same $p$ - $q$-formulas of q.d. $\leq n$. We write $\equiv_{n}$ instead of $\equiv_{n}^{p, q}$ when $p, q$ are clear from the context. The Finiteness Lemma allows to characterize the $n$-equivalence class of a $p$ - $q$-structure by a single $p$ - $q$-formula: 
Corollary 3.3. For all $n \in \mathbb{N}$ and all $p$-q-structure $(\mathcal{M}, \bar{a}, \bar{A})$, there is a formula $\phi \in \Lambda_{n}^{p, q}$ such that for all $p$-q-structure $(\mathcal{N}, \bar{b}, \bar{B})$, we have $(\mathcal{N}, \bar{b}, \bar{B}) \models \phi$ if and only if $(\mathcal{M}, \bar{a}, \bar{B}) \equiv_{n}(\mathcal{N}, \bar{b}, \bar{B})$. Such a $\phi$ is an $n$-characteristic of $(\mathcal{M}, \bar{a}, \bar{B})$.

Moreover, there is a finite set $\Phi_{n}^{p, q} \subseteq \Lambda_{n}^{p, q}$ of $n$-characteristics which contains an $n$-characteristic of each $p$-q-structure.

\subsection{Ehrenfeucht-Fraïssé Games}

Ehrenfeucht-Fraïssé games are a convenient characterization of $\equiv_{n}$-equivalence for languages satisfying the Finiteness Lemma. There are different possible formulations for second-order logic. Our presentation is inspired from [5], which is itself that of [4] adapted to non-full models.

Definition 3.4 (Ehrenfeucht-Fraïssé Games). Given structures $(\mathcal{M}, \bar{a}, \bar{A})$, $(\mathcal{N}, \bar{b}, \bar{B})$ and $n \in \mathbb{N}$, the Ehrenfeucht-Fraïssé Game $\operatorname{EF}_{n}((\mathcal{M}, \bar{a}, \bar{A}),(\mathcal{N}, \bar{b}, \bar{B}))$ is an $n$-round game played between two players called "Spoiler" and "Duplicator".

At each round, Spoiler plays first and chooses either an individual or a predicate in one of the two structures. Duplicator then responds in the other structure by choosing an individual if Spoiler chose an individual or a predicate if Spoiler chose a predicate. After $n$ rounds, Spoiler and Duplicator have build a finite relation

$$
\left\{\left(a_{1}^{\prime}, b_{1}^{\prime}\right), \ldots,\left(a_{p}^{\prime}, b_{p}^{\prime}\right),\left(A_{1}^{\prime}, B_{1}^{\prime}\right), \ldots,\left(A_{q}^{\prime}, B_{q}^{\prime}\right)\right\}
$$

with $n=p+q, \bar{a}^{\prime} \in \mathcal{M}^{\iota}, \bar{b}^{\prime} \in \mathcal{N}^{\iota}, \bar{A}^{\prime} \in \mathcal{M}^{o}$ and $\bar{B}^{\prime} \in \mathcal{N}^{o}$. Then Duplicator wins if and only if $\left(\mathcal{M}, \overline{a a}^{\prime}, \overline{A A}^{\prime}\right) \equiv_{0}\left(\mathcal{N}, \overline{b b}^{\prime}, \overline{B B}^{\prime}\right)$.

Our presentation differs from [5] on the following point. In these works, Duplicator wins if the finishing tuple is a finite partial isomorphism between the two structures. In our case, equality is not a quantifier-free formula, and we take a coarser wining condition based on $\equiv_{0}$-equivalence.

Ehrenfeucht-Fraïssé games characterize $\equiv_{n}$-equivalence:

Theorem 3.5. Given two structures $(\mathcal{M}, \bar{a}, \bar{A})$ and $(\mathcal{N}, \bar{b}, \bar{B})$ and $n \in \mathbb{N}$, Duplicator has a wining strategy in $\operatorname{EF}_{n}((\mathcal{M}, \bar{a}, \bar{A}),(\mathcal{N}, \bar{b}, \bar{B}))$ if and only if $(\mathcal{M}, \bar{a}, \bar{A})$ and $(\mathcal{N}, \bar{b}, \bar{B})$ are $\equiv_{n}$-equivalent.

\section{Finite Sums of Segments}

We now discuss how to restrict structures into segments that can be concatenated. This will be done for second-order linearly ordered structures with correct equality. The Ehrenfeucht-Fraïssé method gives simple proofs that concatenation of segments preserves $\equiv_{n}$-equivalence. This leads to a partial sum operation on $\equiv_{n}$-classes. We follow well-known patterns of Feferman-Vaught Theorems $107 / 5$. 


\subsection{Restrictions and Relativizations}

Segments will be obtained from structures by restrictions and relativizations. The restriction of a structure $\mathcal{M}$ to some non-empty predicate $A \in \mathcal{M}^{o}$ is the structure $\mathcal{M}\left\lceil A\right.$ defined as expected: its individual domain is $\mathcal{M}^{\iota} \cap A$, its predicate domain is $\left\{B \cap A \mid B \in \mathcal{M}^{o}\right\}$ and its relation $<_{\mathcal{M} \uparrow A}$ is the restriction of $<_{\mathcal{M}}$ to $A$ : $<_{\mathcal{M} \uparrow A}:=<_{\mathcal{M}} \cap(A \times A)$. It is convenient to write the individual and predicate domains of $\mathcal{M} \uparrow A$ respectively as $\mathcal{M}^{\iota} \uparrow A$ and $\mathcal{M}^{\circ} \uparrow A$.

Restrictions of Structures with Parameters. We shall also need the less usual restriction of structures with parameters. Let $p, q \in \mathbb{N}$. Consider a structure $\mathcal{M}$ with individual parameters $\bar{a}=a_{1} \ldots a_{p}$ and predicate parameters $\bar{A}=$ $A_{1} \ldots A_{q}$. Let $A \in \mathcal{M}^{o}$ be non-empty and such that $a_{1}, \ldots, a_{p} \in A$. We define the restriction of $(\mathcal{M}, \bar{a}, \bar{A})$ to $A$ to be the structure:

$$
(\mathcal{M}, \bar{a}, \bar{A})\left\lceil A \quad:=\left(\mathcal{M}\left\lceil A, a_{1} \ldots a_{p},\left(A_{1} \cap A\right) \ldots\left(A_{q} \cap A\right)\right)\right.\right.
$$

Relativization of Formulas. An analogous operation can be defined on formulas. Let $\phi$ and $\varphi$ be two formulas with no free variables in common, and let $y$ be a variable not appearing free in $\phi$. The relativization of $\phi$ to $\varphi[y]$, notation $\phi\lceil\varphi[y]$, is defined by induction on $\phi$ as follows:

$$
\begin{aligned}
\phi\lceil\varphi[y] & :=\phi & & \text { if } \phi \text { is atomic } \\
(\phi \vee \psi)\lceil\varphi[y] & :=(\phi\lceil\varphi[y]) \vee(\psi\lceil\varphi[y]) & & \\
(\neg \phi)\lceil\varphi[y] & :=\neg(\phi\lceil\varphi[y]) & & \\
(\exists X \phi)\lceil\varphi[y] & :=\exists X(\phi\lceil\varphi[y]) & & \text { if } X \notin \operatorname{FV}(\varphi) \\
(\exists x \phi)\lceil\varphi[y] & :=\exists x(\varphi[x / y] \wedge \phi\lceil\varphi[y]) & & \text { if } x \notin \operatorname{FV}(\varphi) \cup\{y\}
\end{aligned}
$$

If $(\phi, \nu)$ is a formula with parameters in a structure $\mathcal{M}$, and if $A \in \mathcal{M}^{o}$ contains all individual parameters of $\phi$, then $(\phi, \nu)\lceil A$ is defined as $((\phi \uparrow(X x)[x]), \nu[A / X])$ where $X, x \notin \mathrm{FV}(\phi, \nu)$.

The Transfer Property. We now check that restriction and relativization are equivalent w.r.t. satisfaction. This in particular implies that restriction preserves the comprehension scheme: $\mathcal{M} \uparrow A$ is second-order if $\mathcal{M}$ is second-order.

Proposition 4.1 (Transfer). Let $p, q \in \mathbb{N}$ and $(\mathcal{M}, \bar{a}, \bar{A})$ be a p-q-structure. Let $\varphi$ be a formula with parameters in $\mathcal{M}$ and whose free variables are disjoint from $\left\{x_{1}, \ldots, x_{p}, X_{1}, \ldots, X_{q}\right\}$. Given $x_{0} \notin\left\{x_{1}, \ldots, x_{p}\right\}$, let $A \in \mathcal{M}^{o}$ be nonempty and such that $(\mathcal{M}, \bar{a}, \bar{A}) \models \forall x\left(A x \longleftrightarrow \varphi\left[x / x_{0}\right]\right)$. Assume that $\bar{a} \in A$.

Let $\phi$ be a formula with $\mathrm{FV}(\phi) \subseteq\left\{x_{1}, \ldots, x_{p}, X_{1}, \ldots, X_{q}\right\}$. Then we have $(\mathcal{M}, \bar{a}, \bar{A})\left\lceil A \models \phi\right.$ if and only if $(\mathcal{M}, \bar{a}, \bar{A}) \models \phi\left\lceil\varphi\left[x_{0}\right]\right.$.

\subsection{Finite Sums of Segments}

A segment of a structure $\mathcal{M}$ is a predicate of the form

$$
[a, b):=\left\{c \in \mathcal{M}^{\iota} \mid a \leq_{\mathcal{M}} c<_{\mathcal{M}} b\right\} \quad \text { where } a<_{\mathcal{M}} b
$$


We write $[-, b)$ for $[a, b)$ if $\mathcal{M}$ is linearly ordered with least element $a$. Two consecutive segments $(\mathcal{M}, \bar{a}, \bar{A}) \uparrow[a, b)$ and $(\mathcal{M}, \bar{b}, \bar{A}) \uparrow[b, c)$ can be concatenated to $(\mathcal{M}, \bar{a} \bar{b}, \bar{A}) \uparrow[a, c)$. Using the Ehrenfeucht-Fraïssé method, it is easy to show that concatenation of segments preserves $\equiv_{n}$-equivalence.

Similar operations have already been defined for full models (see e.g. [10]) as well as for Henkin models [5]. Our operation differs from [5] in the treatment of predicate parameters: since we only need the concatenation of consecutive segments which are restrictions of the same structure $\mathcal{M}$, we can share the predicate parameters in the two components. This simplifies both the statement and the proof of the Lemma.

Lemma 4.2 (Finite Sums of Segments). Consider two second-order linearly ordered structures $\mathcal{M}$ and $\mathcal{N}$, both with correct equality. Let $n \in \mathbb{N}$.

$$
\begin{aligned}
\text { If } \quad(\mathcal{M}, \bar{a}, \bar{A}) \uparrow\left[t_{0}, t_{1}\right) & \equiv_{n}(\mathcal{N}, \bar{b}, \bar{B}) \uparrow\left[u_{0}, u_{1}\right) \\
\text { and }\left(\mathcal{M}, \bar{a}^{\prime}, \bar{A}\right) \uparrow\left[t_{1}, t_{2}\right) & \equiv_{n}\left(\mathcal{N}, \bar{b}^{\prime}, \bar{B}\right) \uparrow\left[u_{1}, u_{2}\right) \\
\text { then }\left(\mathcal{M}, \overline{a a}^{\prime}, \bar{A}\right) \uparrow\left[t_{0}, t_{2}\right) & \equiv_{n}\left(\mathcal{N}, \overline{b b}^{\prime}, \bar{B}\right) \uparrow\left[u_{0}, u_{2}\right) .
\end{aligned}
$$

\section{Completeness of $\mathrm{MSO}^{\omega}$ w.r.t. the Standard Model}

In this section, we present a proof of Theorem 2.8. We use an infinitary version of the Finite Sums Lemma which is discussed in Section 6 .

We actually prove the following formulation of Theorem 2.8 .

Theorem 5.1. Let $\mathcal{M}$ be a model of $M_{S O}{ }^{\omega}$. For all $n \in \mathbb{N}$ we have $\mathcal{M} \equiv_{n} \mathrm{~N}$.

Doets' Lemma. Our way to Theorem 5.1 starts from the simple but crucial observation that bounded segments of models of $\mathrm{MSO}^{\omega}$ are $\equiv_{n}$-equivalent to finite linear orders. To our knowledge, this is due to K. Doets [3] for the $\Pi_{1}^{1}$ case (first-order logic with universal prenex quantification on predicates). Recall that a bounded segment of an arbitrary model of $\mathrm{MSO}^{\omega}$ may not be finite (see Remark 2.91).

In our context, a finite linear order is a structure of the form $\mathrm{N} \uparrow\left[m_{0}, m_{1}\right)$ with $m_{0}<m_{1} \in \mathbb{N}$. Note that if $m_{1}-m_{0}=k_{1}-k_{0}$ (where $m_{0}<m_{1}$ and $k_{0}<k_{1}$ ), then $\mathrm{N} \uparrow\left[m_{0}, m_{1}\right) \equiv_{n} \mathrm{~N} \uparrow\left[k_{0}, k_{1}\right)$ for all $n \in \mathbb{N}$.

Lemma 5.2 (Doets' Lemma). Let $\mathcal{M}$ be a model of $M O^{\omega}$ and $n \in \mathbb{N}$. For all $a<_{\mathcal{M}} b$, there is a finite linear order $\mathcal{L}$ such that $\mathcal{M} \uparrow[a, b) \equiv_{n} \mathcal{L}$.

Ramseyan Factorizations. Let $\mathcal{M}$ be a model of $\mathrm{MSO}^{\omega}$. In order to obtain $\mathcal{M} \equiv_{n}$ $\mathrm{N}$ from Doets' Lemma 5.2 , we would like to perform a kind of infinite sum of the $(\mathcal{M} \uparrow[a, b))_{a<\mathcal{M} b}$. We rely on a weak form of Ramsey's theorem which is similar to the usual Ramseyan factorizations of infinite words discussed e.g. in [8].

Recall from Corollary 3.3 that if $\mathcal{M}$ is a linearly ordered second-order structure with correct equality, then for all $n \in \mathbb{N}$ and all $a<_{\mathcal{M}} b$, there is a $\phi \in \Phi_{n}^{0,0}$ such that $\mathcal{M} \models \phi\lceil[a, b)$. We say that $\mathcal{M}$ has Ramseyan factorizations if there is 
$\phi \in \Phi_{n}^{0,0}$ and an unbounded $U \in \mathcal{M}^{o}$ which is homogeneous for $\phi$. We actually need a slightly stronger statement involving formulas with predicate parameters.

Given a structure $\mathcal{M}$ and a predicate $U \in \mathcal{M}^{o}$, we let $[U]^{2} \subseteq \mathcal{M}^{\iota} \times \mathcal{M}^{\iota}$ be the set of pairs $(a, b) \in U \times U$ such that $a<_{\mathcal{M}} b$.

Theorem 5.3 (Ramseyan Factorizations). Let $\mathcal{M}$ be a model of $M_{S} \mathrm{O}^{\omega}$ and let $n, q \in \mathbb{N}$. Given $A_{1}, \ldots, A_{q} \in \mathcal{M}^{o}$ and an unbounded $U \in \mathcal{M}^{o}$, there is an unbounded predicate $V \subseteq U$ and $a \phi \in \Phi_{n}^{0, q}$ such that for all $(a, b) \in[V]^{2}$ we have $(\mathcal{M}, \bar{A}) \models \phi \uparrow[a, b)$.

Theorem 5.3 follows from Theorem I.1.c.3 of 11. See also the full version of this paper. Together with Doets' Lemma, we obtain:

Corollary 5.4. Let $\mathcal{M}$ be a model of $M S O^{\omega}$ and $n \in \mathbb{N}$. There is an unbounded $U \in \mathcal{M}^{\circ}$ and a finite linear order $\mathcal{L}$ such that for all $(a, b) \in[U]^{2}$ we have $\mathcal{M} \uparrow[a, b) \equiv_{n} \mathcal{L}$.

Infinite Fusion. Let $\mathcal{M}$ be a model of $\mathrm{MSO}^{\omega}$ and $n \in \mathbb{N}$. Using Corollary 5.4 and Doets' Lemma 5.2 we arrive at the following point: There are unbounded $U \in \mathcal{M}^{o}$ and $V \in \mathcal{P}(\mathbb{N})$ together with $u \in U$ and $v \in V$ such that

$$
\mathcal{M} \uparrow[-, u) \equiv_{n} \mathrm{~N} \uparrow[-, v)
$$

and for all $\left(u_{0}, u_{1}\right) \in[U]^{2}$ and all $\left(v_{0}, v_{1}\right) \in[V]^{2}$

$$
\mathcal{M} \uparrow\left[u_{0}, u_{1}\right) \equiv_{n} \mathrm{~N} \uparrow\left[v_{0}, v_{1}\right)
$$

We can conclude that $\mathcal{M} \equiv_{n} \mathrm{~N}$ from these assumptions thanks to the Infinite Fusion Lemma 6.2. We state and prove it in Section 6, and this will achieve the proof of Theorem 5.1 .

\section{The Infinite Fusion Lemma}

In this section, we state and prove the Infinite Fusion Lemma. Besides Ramseyan factorizations (already discussed in Section 5), we shall also use a weak form of the axiom of choice which is called Splicing in [11.

\subsection{Splicing}

We discuss the Splicing Theorem of [11] and one of its corollary that we actually use in the Infinite Fusion Lemma.

Let $\mathcal{M}$ be a model of $\mathrm{MSO}^{\omega}$ and $U \in \mathcal{M}^{o}$. Individuals $a, b \in \mathcal{M}^{\iota}$ are consecutive in $U$ if $a, b \in U, a<_{\mathcal{M}} b$ and there is no $c \in U$ such that $a<_{\mathcal{M}} c<_{\mathcal{M}} b$.

The Splicing Theorem is the following: Given a formula $(\exists X \phi)$ with predicate parameters in $\mathcal{M}$, if for all $a, b$ consecutive in $U$ we have $\mathcal{M} \models \exists X \phi\lceil[a, b)$, then there is a predicate $A \in \mathcal{M}^{o}$ such that for all $a, b$ consecutive in $U$ we have $\mathcal{M} \models \phi[A / X] \uparrow[a, b)$. This is Theorem I.5.b.1 of [11]. 
For the Infinite Fusion Lemma, we shall use a variant of Splicing that we call Idempotent Splicing. The main difference is that we need to obtain a predicate $A \in \mathcal{M}^{o}$ which is correct for all $(a, b) \in[U]^{2}$, and not just the consecutive ones. On the other hand, we only need it for those $\equiv_{n}^{q}$-characteristics which moreover define an idempotent coloring.

Proposition 6.1 (Idempotent Splicing). Let $\mathcal{M}$ be a model of $M S O^{\omega}$ and let $n, q \in \mathbb{N}$.

Given and $A_{1}, \ldots, A_{q} \in \mathcal{M}^{o}$, let $\phi \in \Phi_{n}^{0,(q+1)}$ and $U \in \mathcal{M}^{o}$ be such that

(i) $(\mathcal{M}, \bar{A}) \models \exists X \phi\left[X / X_{q+1}\right]\left\lceil[a, b)\right.$ for all $(a, b) \in[U]^{2}$, and

(ii) there is a second-order linearly ordered 0 - $(q+1)$-structure with correct equality $(\mathcal{N},, \bar{B} B)$ and $b_{0}<_{\mathcal{N}} b_{1}<_{\mathcal{N}} b_{3}$ such that $\phi$ holds in $(\mathcal{N},, \bar{B} B) \uparrow\left[b_{0}, b_{2}\right),(\mathcal{N}, \bar{B} B) \uparrow\left[b_{0}, b_{1}\right)$ and $(\mathcal{N}, \bar{B} B) \uparrow\left[b_{1}, b_{2}\right)$.

Then there is a predicate $A \in \mathcal{M}^{o}$ such that for all $(a, b) \in[U]^{2}$ we have $(\mathcal{M}, \bar{A} A) \models \phi \uparrow[a, b)$.

In Proposition 6.1 above, condition (ii) is actually the premise of the Splicing Theorem. Condition (iii) intuitively says that $\phi$ defines an idempotent coloring. We give more details on Splicing in the full version of the paper.

\subsection{Infinite Fusion}

As usual with the Ehrenfeucht-Fraïssé method, we perform an induction on the quantifier depth of formulas. This to consider structures with parameters.

Lemma 6.2 (Infinite Fusion). Let $\mathcal{M}$ and $\mathcal{N}$ be models of $M S O^{\omega}$. Let $n \in \mathbb{N}$.

Let $U \in \mathcal{M}^{\circ}$ and $V \in \mathcal{N}^{o}$ be unbounded, and assume that their respective least elements $u$ and $v$ are not the least elements of respectively $\mathcal{M}^{\iota}$ and $\mathcal{N}^{\iota}$.

Let $\bar{a} \in \mathcal{M}^{\iota} \uparrow[-, u), \bar{b} \in \mathcal{N}^{\iota} \uparrow[-, v)$ both of length $p \in \mathbb{N}$ and $\bar{A} \in \mathcal{M}^{o}, \bar{B} \in \mathcal{N}^{o}$ both of length $q \in \mathbb{N}$. Assume that

$$
(\mathcal{M}, \bar{a}, \bar{A})\left\lceil[-, u) \equiv_{n}(\mathcal{N}, \bar{b}, \bar{B})\lceil[-, v)\right.
$$

and that for all $\left(u_{0}, u_{1}\right) \in[U]^{2}$ and all $\left(v_{0}, v_{1}\right) \in[V]^{2}$ we have

$$
(\mathcal{M}, \bar{A}) \uparrow\left[u_{0}, u_{1}\right) \equiv_{n}(\mathcal{N}, \bar{B})\left\lceil\left[v_{0}, v_{1}\right)\right.
$$

Then $(\mathcal{M}, \bar{a}, \bar{A}) \equiv_{n}(\mathcal{N}, \bar{b}, \bar{B})$.

Using Lemma 6.2, we can achieve the proof of Theorem 5.1 as follows. Let $\mathcal{M}$ be a model of $\mathrm{MSO}^{\omega}$ and let $n \in \mathbb{N}$. By Corollary 5.4 there is a finite linear order $\mathcal{L}$ and an unbounded predicate $U \in \mathcal{M}^{o}$ such that $\mathcal{M} \uparrow[a, b) \equiv_{n} \mathcal{L}$ for all $(a, b) \in[U]^{2}$. Since $\mathcal{M}$ is a second-order linearly ordered structure with correct equality, we can moreover assume that the least element $u$ of $U$ is not the least element of $\mathcal{M}$. By Doets' Lemma 5.2, the segment $\mathcal{M}[-, u)$ is $\equiv_{n}$-equivalent to $\mathrm{N} \uparrow[-, v)$ for some $v>0$. We thus obtain an unbounded set $V \in \mathcal{P}(\mathbb{N})$ with least element $v$, and such that $\mathcal{M} \uparrow[-, u) \equiv_{n} \mathrm{~N} \uparrow[-, v)$ and $\mathcal{M} \uparrow\left[u_{0}, u_{1}\right) \equiv_{n} \mathrm{~N} \uparrow\left[v_{0}, v_{1}\right)$ for all $\left(u_{0}, u_{1}\right) \in[U]^{2}$ and all $\left(v_{0}, v_{1}\right) \in[V]^{2}$. We conclude $\mathcal{M} \equiv_{n} \mathrm{~N}$ by Lemma 6.2 ,

The rest of this section is devoted to the proof of Lemma 6.2. We reason by (external) induction on $n \in \mathbb{N}$. 
Base Case $(\boldsymbol{n}=\mathbf{0})$. We just have to show that $(\mathcal{M}, \bar{a}, \bar{A})$ and $(\mathcal{N}, \bar{b}, \bar{B})$ agree on atomic formulas $\phi$ with individual variables in $\left\{x_{1}, \ldots, x_{p}\right\}$ and predicate variables in $\left\{X_{1}, \ldots, X_{q}\right\}$. We only detail the case of $x_{i}<x_{j}$, that of $X_{i} x_{j}$ being similar.

Since $a_{i}, a_{j}<_{\mathcal{M}} u$, the formula $\left(x_{i}<x_{j}\right)$ holds in $(\mathcal{M}, \bar{a}, \bar{A})$ if and only if it holds in $(\mathcal{M}, \bar{a}, \bar{A}) \uparrow[-, u)$. The same holds for $\mathcal{N}$, and we are done since $(\mathcal{M}, \bar{a}, \bar{A}) \uparrow[-, u) \equiv_{0}(\mathcal{N}, \bar{b}, \bar{B}) \uparrow[-, v)$.

Inductive Step. We now consider the inductive step: we show the property for $n+1$ assuming it for $n$. Using Theorem 3.5. we consider the different possible moves of Spoiler, and then build the answer of Duplicator.

Spoiler plays an individual, say $a \in \mathcal{M}^{\iota}$. Since $U$ is unbounded, there is $u^{\prime} \in U$ strictly greater than $a$. Also using the unboundedness of $V$, let $v^{\prime} \in V$ be strictly greater than $v$.

We have $(\mathcal{M}, \bar{a}, \bar{A}) \uparrow\left[-, u^{\prime}\right) \equiv_{n+1}(\mathcal{N}, \bar{b}, \bar{B}) \uparrow\left[-, v^{\prime}\right)$ thanks to the Finite Sums Lemma 4.2 applied to the assumptions $(\mathcal{M}, \bar{a}, \bar{A}) \uparrow[-, u) \equiv_{n+1}(\mathcal{N}, \bar{b}, \bar{B}) \uparrow[-, v)$ and $(\mathcal{M}, \bar{A}) \uparrow\left[u, u^{\prime}\right) \equiv_{n+1}(\mathcal{N}, \bar{B}) \uparrow\left[v, v^{\prime}\right)$. Now, by Theorem 3.5 there is some $b \in \mathcal{N}^{\iota} \uparrow\left[-, v^{\prime}\right)$ such that $(\mathcal{M}, \bar{a} a, \bar{A}) \uparrow\left[-, u^{\prime}\right) \equiv_{n}(\mathcal{N}, \bar{b} b, \bar{B}) \uparrow\left[-, v^{\prime}\right)$. The predicates $U^{\prime}:=\left\{s \in U \mid s \geq_{\mathcal{M}} u^{\prime}\right\}$ and $V^{\prime}:=\left\{t \in V \mid t \geq_{\mathcal{N}} v^{\prime}\right\}$ are both unbounded. For all $\left(u_{0}, u_{1}\right) \in\left[U^{\prime}\right]^{2},\left(v_{0}, v_{1}\right) \in\left[V^{\prime}\right]^{2}$, we have $(\mathcal{M}, \bar{A}) \uparrow\left[u_{0}, u_{1}\right) \equiv_{n}$ $(\mathcal{N}, \bar{B})\left\lceil\left[v_{0}, v_{1}\right)\right.$. Moreover, since $\mathcal{M}$ and $\mathcal{N}$ are both linearly ordered and with correct equality, $u^{\prime}$ and $v^{\prime}$ are the least elements of respectively $U^{\prime}$ and $V^{\prime}$. We can thus conclude by induction hypothesis.

Spoiler plays a predicate, say $A \in \mathcal{M}^{o}$. Since $\mathcal{M}$ has Ramseyan factorizations (Theorem [5.3) we get an unbounded predicate $U^{\prime} \subseteq U$ and an $n$-characteristic $\phi \in \Phi_{n}^{0, q+1}$ such that for all $\left(u_{0}, u_{1}\right) \in\left[U^{\prime}\right]^{2}$ we have $(\mathcal{M}, \bar{A} A) \models \phi \uparrow\left[u_{0}, u_{1}\right)$, i.e. $(\mathcal{M}, \bar{A} A)\left\lceil\left[u_{0}, u_{1}\right) \models \phi\right.$ thanks to the Transfer Property (Proposition 4.1). Since $U^{\prime}$ is unbounded and since on the other hand $\mathcal{M}$ is a second-order linearly ordered structure with correct equality, we can assume that $U^{\prime}$ has a least element $u^{\prime}$.

We now claim that for all $\left(v_{0}, v_{1}\right) \in[V]^{2}$ we have $(\mathcal{N}, \bar{B}) \models(\exists X \phi) \uparrow\left[v_{0}, v_{1}\right)$.

- Proof (of the claim). Fix $\left(u_{0}, u_{1}\right) \in\left[U^{\prime}\right]^{2} \subseteq[U]^{2}$. For all $\left(v_{0}, v_{1}\right) \in[V]^{2}$, since by assumption $(\mathcal{N}, \bar{B})\left\lceil\left[v_{0}, v_{1}\right) \equiv_{n+1}(\mathcal{M}, \bar{A}) \uparrow\left[u_{0}, u_{1}\right)\right.$, by Theorem 3.5 there is some $B^{v_{0}, v_{1}} \in \mathcal{N}^{o}$ such that $\left(\mathcal{N}, \bar{B} B^{v_{0}, v_{1}}\right)\left\lceil\left[v_{0}, v_{1}\right) \equiv_{n}(\mathcal{M}, \bar{A} A) \uparrow\left[u_{0}, u_{1}\right)\right.$, hence $\left(\mathcal{N}, \bar{B} B^{v_{0}, v_{1}}\right) \uparrow\left[v_{0}, v_{1}\right) \models \phi$.

By Proposition 6.1 (Idempotent Splicing), there is a predicate $B \in \mathcal{N}^{\circ}$ such that $(\mathcal{N},, \bar{B} B) \models \phi \uparrow\left[v_{0}, v_{1}\right)$ for all $\left(v_{0}, v_{1}\right) \in[V]^{2}$. Note that condition (iii) of Proposition 6.1 is satisfied with $(\mathcal{M},, \bar{A} A)$ and any $u_{0}^{\prime}<_{\mathcal{M}} u_{1}^{\prime}<_{\mathcal{M}} u_{2}^{\prime}$ in the unbounded predicate $U^{\prime}$.

We now build Duplicator's response to $A \in \mathcal{M}^{o}$. We have to take care of the initial segment $(\mathcal{M}, \bar{A} A) \uparrow\left[-, u^{\prime}\right)$. Using the unboundedness of $V$, let $v^{\prime} \in V$ be strictly greater than $v$. Reasoning as above, we get $(\mathcal{M}, \bar{a}, \bar{A}) \uparrow\left[-, u^{\prime}\right) \equiv_{n+1}$ 
$(\mathcal{N}, \bar{b}, \bar{B}) \uparrow\left[-, v^{\prime}\right)$ using the Finite Sums Lemma 4.2, Let $V^{\prime}:=\left\{c \in V \mid v^{\prime} \leq_{\mathcal{N}}\right.$ $c\}$. By Theorem 3.5, there is some $B^{\prime} \in \mathcal{N}^{o}$ such that $\left(\mathcal{N}, \bar{B} B^{\prime}\right) \uparrow\left[-, v^{\prime}\right) \equiv_{n}$ $(\mathcal{M}, \bar{A} A) \uparrow\left[-, u^{\prime}\right)$. Since $\mathcal{N}$ is second-order, let $B^{\prime \prime}:=B^{\prime} \uparrow\left[-, v^{\prime}\right) \cup B \uparrow\left[v^{\prime},-\right)$, where $\left[v^{\prime},-\right):=\left\{c \in \mathcal{N}^{\iota} \mid v^{\prime} \leq_{\mathcal{N}} c\right\}$. Now, $\left(\mathcal{N}, \bar{b}, \bar{B} B^{\prime \prime}\right)$ (together with $\left.V^{\prime}\right)$ satisfies the premise of the induction hypothesis and we are done.

\section{Conclusion}

We gave a model-theoretic proof of Siefkes' completeness result for MSO ${ }^{\omega}[11$. It is based on Ramsey's Theorem for additive colorings, with constructions reminiscent from algebraic approaches to $\omega$-rational languages [8]. Further works will begin by clarifying these relationships. An interesting question is the prooftheoretic analysis of $\mathrm{MSO}^{\omega}$. The algebraic approach to parity conditions [8] can be interesting in this perspective. An other direction is the completeness of MSO on infinite trees, and the comparison with Walukiewicz's completeness result for the $\mu$-calculus $[12$.

\section{References}

1. Boolos, G.S., Burgess, J.P., Jeffrey, R.C.: Computability and Logic, 5th edn. Cambridge University Press (2007)

2. Büchi, J.R.: On a Decision Methond in Restricted Second-Order Arithmetic. In: Nagel, E., et al. (eds.) Logic, Methodology and Philosophy of Science. Proc. 1960 Intern. Congr., pp. 1-11. Stanford Univ. Press (1962)

3. Doets, K.: Monadic $\Pi_{1}^{1}$-Theories of $\Pi_{1}^{1}$-Properties. Notre Dame Journal of Formal Logic 30(2), 224-240 (1989)

4. Ebbinghaus, H.-D., Flum, J.: Finite Model Theory, 2nd edn. Springer Monographs in Mathematics. Springer (1999)

5. Gheerbrant, A., ten Cate, B.: Complete Axiomatizations of MSO, FO(TC $\left.{ }^{1}\right)$ and FO $\left(\right.$ LFP $\left.^{1}\right)$ on Finite Trees. In: Artemov, S., Nerode, A. (eds.) LFCS 2009. LNCS, vol. 5407, pp. 180-196. Springer, Heidelberg (2008)

6. Grädel, E., Thomas, W., Wilke, T. (eds.): Automata, Logics, and Infinite Games: A Guide to Current Research. LNCS, vol. 2500. Springer, Heidelberg (2002)

7. Gurevich, Y.: Monadic Second-Order Theories. In: Barwise, J., Feferman, S. (eds.) Model-Theoretical Logics. Perspective in Mathematical Logic, pp. 479-506. Springer (1985)

8. Perrin, D., Pin, J.-É.: Infinite Words: Automata, Semigroups, Logic and Games. Pure and Applied Mathematics. Elsevier (2004)

9. Shapiro, S.: Foundations without Foundationalism: A Case for Second-Order Logic. Oxford University Press (1991)

10. Shelah, S.: The Monadic Theory of Order. The Annals of Mathematics, Second Series 102(3), 379-419 (1975)

11. Siefkes, D.: Decidable Theories I: Büchi's Monadic Second Order Successor Arithmetic. LNM, vol. 120. Springer (1970)

12. Walukiewicz, I.: Completeness of Kozen's Axiomatisation of the Propositional $\mu$ Calculus. Information and Computation 157(1-2), 142-182 (2000) 